\title{
AN EMPIRICAL VERSUS ANALYTICAL APPROACH FOR MODELLING BIOFILM GROWTH IN BIOFILTERS
}

\author{
JACOLINE VAN JAARSVELD \& SONIA WOUDBERG \\ Department of Mathematical Sciences, Stellenbosch University, South Africa
}

\begin{abstract}
In this study, experimental pressure drop data obtained for a biofilter containing expanded schist as packing material is used in order to characterize the packed bed properties. The biofilter was operated over a period of 106 days. Two modelling approaches were used: an empirical and an analytical approach. In the empirical approach the pressure drop prediction of the modified-Macdonald model, Representative Unit Cell (RUC) model and the model of Comiti and Renaud are used to determine the biofilm affected porosity, specific surface area and tortuosity. This was done by using Excel ${ }^{\circledR}$ Solver which is based on an optimization method. In the analytical approach the biofilm thickness is incorporated into the RUC model and used to predict the specific surface area, as well as the pressure drop. The effect of particle sphericity on the pressure drop is also investigated. The results obtained by the two modelling approaches are compared and analysed. The suitability of the three models used in the biofilter analysis is also determined based on the accuracy of predictions compared to the experimental data.
\end{abstract}

Keywords: granular, porous media, pressure drop, modelling, biofilm, biofilter, porosity, specific surface area, tortuosity, sphericity.

\section{INTRODUCTION}

Hydrogen sulphide is an example of a poisonous gas being emitted into the atmosphere on a daily basis. The environmentally friendly process of biofiltration can remove such poisonous gasses from an air stream.

A biofilter consists of a packed bed containing packing material and thin layers of moisture, known as biofilm. The packing material, which in this case is known as schist, acts like a filter when a polluted air stream passes through the packed bed. When a poisonous gas, consisting of biodegradable pollutants, is slowly being pumped through the biofilter, the biofilm is responsible for biologically oxidizing the polluted gas [1]-[3].

Dumont et al. [4] applied three porous media models to experimental pressure drop measurements in order to assess the development of the biofilm thickness over time in three different biofilters. The measured values include the pressure drop, superficial velocity, porosity on day 0 and the particle diameter. The days of biofilter operation on which the latter data was recorded are: Days 0, 19, 39, 57, 71, 92 and 106. The three models used are the modified-Macdonald (MM) model, the Comiti and Renaud (CR) model and the Representative Unit Cell (RUC) model. An empirical approach was followed in which Excel $^{\circledR}$ Solver was used as optimization tool. Woudberg et al. [5] used the analytical approach based on the RUC model in order to predict the pressure drop and the biofilm affected specific surface area (SSA) as a function of biofilm thickness.

The objective of this study is to analyse the two modelling procedures in terms of the advantages and limitations of each approach. The suitability of the three models used in the analysis is also determined based on the accuracy of predictions compared to the experimental data. 


\section{MODELS FROM THE LITERATURE}

The MM model is based on an adaptation of the empirical Ergun equation, for which the pressure gradient is given [6], i.e.,

$$
-\frac{d p}{d x}=180 \frac{\mu q}{D_{p}^{2} \varphi^{2}} \frac{(1-\varepsilon)^{2}}{\varepsilon^{3.6}}+B \frac{\rho q^{2}}{D_{p} \varphi} \frac{(1-\varepsilon)}{\varepsilon^{3.6}}
$$

In eqn (1) the sphericity is denoted by $\varphi, q$ denotes the magnitude of the superficial velocity and $\varepsilon$ is the porosity. The particle diameter is denoted by $D_{p}$. The gas properties, i.e. $\rho$ a n d $\mu$, represent the density and the viscosity, respectively. The value of the coefficient $B$ is dependent on surface roughness and may vary between 1.8 and 4.0 [6]. The capillary-tube model of Comiti and Renaud [7] is given in eqn (2):

$$
-\frac{d p}{d x}=2 \tau^{2} \mu a_{v d}^{2} q \frac{(1-\varepsilon)^{2}}{\varepsilon^{3}}+0.0968 \tau^{3} \rho q^{2} a_{v d} \frac{(1-\varepsilon)}{\varepsilon^{3}},
$$

where $\tau$ is known as the tortuosity and $a_{v d}$ denotes the SSA, without taking biofilm growth into consideration. The RUC model of Woudberg et al. [5] is given in eqn (3):

$$
-\frac{d p}{d x}=25.4 \frac{\mu q}{D_{p}^{2} \varphi^{2}} \frac{(1-\varepsilon)^{4 / 3}}{\left(1-(1-\varepsilon)^{1 / 3}\right)\left(1-(1-\varepsilon)^{2 / 3}\right)^{2}}+\beta c_{d} \frac{\rho q^{2}}{D_{p} \varphi} \frac{(1-\varepsilon)}{\varepsilon\left(1-(1-\varepsilon)^{2 / 3}\right)^{2}},
$$

in which $\varphi=2$ and $\beta=0.85$ [4]. A value of 1.9 is assigned to the form drag coefficient $c_{d}$, since it provided exact correspondence to the higher Reynolds number term of the Macdonald eqn [8].

\section{EMPIRICAL MODELLING APPROACH}

Dumont et al. [4] used an empirical approach to estimate the porosity values on each day of biofilter operation as well as to predict the corresponding biofilm thickness values by making use of measured pressure drop values. The MM model, Excel ${ }^{\circledR}$ Solver and the sum of the squared residuals were used to determine the value of $\varphi$. The value of 0.85 obtained is consistent with the value used by Macdonald et al. [9]. This value was also used in the RUC model.

The MM model (eqn (1)) contains two unknowns that were optimized with Excel ${ }^{\circledR}$ Solver, i.e. $\varepsilon$ and $B$. In the CR model (eqn (2)) the three unknowns, i.e. $\varepsilon, a_{v d}$, and $\tau$ were optimized. In the RUC model (eqn (3)) only $\varepsilon$ needed to be optimized.

For every model and every day on which pressure drop data was recorded, the experimentally measured porosity value was used as an initial guess for the porosity. After comparing the $\varepsilon$-values determined by the three models, with the measured $\varepsilon$-values, Dumont et al. [4] found that the CR model cannot be used to predict the porosity of a packed bed, based on significant discrepancies. The CR model was therefore excluded from predicting biofilm thickness values. The RUC model somewhat underestimated the measured porosity values and the MM model gave remarkable results when compared to the experimental values.

By making use of the predicted $\varepsilon$-values obtained with the MM model, Dumont et al. [4] determined the biofilm thickness, $L_{f}$, by solving the following equation of Alonso et al. [1] for the biofilm affected porosity, $\varepsilon_{f}$, i.e.:

$$
\varepsilon_{f}=1-\left(1-\varepsilon_{0}\right)\left[\left(1+\frac{2 L_{f}}{\varphi D_{p}}\right)^{3}-\frac{n}{4}\left(\frac{2 L_{f}}{\varphi D_{p}}\right)^{2}\left(\frac{4 L_{f}}{\varphi D_{p}}+3\right)\right],
$$


where $D_{p}=0.01$ and $n$ denotes the coordination number. The coordination number for a close, random type of packing is related to the initial porosity, $\varepsilon_{0}$, as follows [1]:

$$
\varepsilon_{0}=1.072-0.1193 n+0.004312 n^{2}
$$

For $\varepsilon_{0}=0.4230$ a value of 7 is obtained for $n$. Finally, the biofilm affected SSA, $a_{f}$, was determined from the following eqn [1]:

$$
a_{f}=\frac{3\left(1-\varepsilon_{0}\right)}{\varphi D_{p}}\left[\left(1+\frac{2 L_{f}}{\phi D_{p}}\right)\left((2-n) \frac{2 L_{f}}{\phi D_{p}}+2\right)\right]
$$

\section{ANALYTICAL MODELLING APPROACH}

In the analytical approach of Woudberg et al. [5] the $L_{f}$-values were also obtained from eqn. 4 by making use of the measured porosity values for each day. The $a_{f}$-values for each day on which measurements were recorded were determined from eqn (6). The latter equation was incorporated into eqn (3), yielding:

$$
\begin{gathered}
-\frac{1}{q} \frac{d p}{d x}=\frac{25.4\left(1-\varepsilon_{f}\right)^{4 / 3} \mu a_{f}^{2}}{9\left(1-\varepsilon_{0}\right)^{2}\left(1-\left(1-\varepsilon_{f}\right)^{1 / 3}\right)\left(1-\left(1-\varepsilon_{f}\right)^{2 / 3}\right)^{2}}\left[\left(1+\frac{L_{f}}{\varphi R}\right)\left((2-n) \frac{L_{f}}{\varphi R}+2\right)\right]^{-2}+ \\
\frac{\beta\left(1-\varepsilon_{f}\right) c_{d} \rho q a_{f}}{6 \varepsilon_{f}\left(1-\varepsilon_{0}\right)\left(1-\left(1-\varepsilon_{f}\right)^{2 / 3}\right)^{2}}\left[\left(1+\frac{L_{f}}{\varphi R}\right)\left((2-n) \frac{L_{f}}{\varphi R}+2\right)\right]^{-1}
\end{gathered}
$$

to subsequently predict the pressure gradient divided by the magnitude of $\vec{q}$ for each day of the experiment. The parameter, $R$, is defined as that radius of the packing material particle.

Alternatively, the $a_{f}$-values were obtained from eqn (7) by making use of the measured pressure drop, superficial velocity and porosity values. After obtaining the $a_{f}$-values corresponding to the seven different superficial velocity and pressure drop values, the average $a_{f}$-values were calculated for each day. Both methods for calculating the SSA values were found to be adequate, based on the same order of magnitude values that were obtained.

\section{ANALYSIS OF THE TWO MODELLING APPROACHES}

In addition to the value of $\varphi=0.85$ used by Dumont et al. [4], an average $\varphi$-value is calculated in this study for the analytical modelling approach using

$$
\varphi=\frac{\pi^{3}\left(6 V_{p}\right)^{\frac{2}{3}}}{A_{p}} \frac{\pi^{3}\left(8 \pi\left(R+L_{f}\right)^{3}+8 \pi n R^{3}+24 \pi n L_{f} R^{2}+12 \pi n L_{f}{ }^{2} R\right)^{\frac{2}{3}}}{4 \pi\left(R+L_{f}\right)^{2}+4 \pi n R^{2}+4 \pi n L_{f} R},
$$

yielding a value of 0.5 .

The pressure gradient predicted for day 39 with both the MM model and the RUC model with different values of $\varphi$, are compared to the experimental data in Fig. 1.

The MM model (with $B=4.0$ ) and the RUC model (using $L_{f}$ ) with $\varphi=0.85$ capture the experimental data in between. The MM model slightly overpredicts the data whereas the RUC model slightly under-predicts the data. The RUC model (using $L_{f}$ ) with $\varphi=0.50$ and $\varphi=0.85$ also captures the experimental data. The over- and underprediction of the RUC model illustrates the significant effect of the value used for $\varphi$ in the model prediction. 


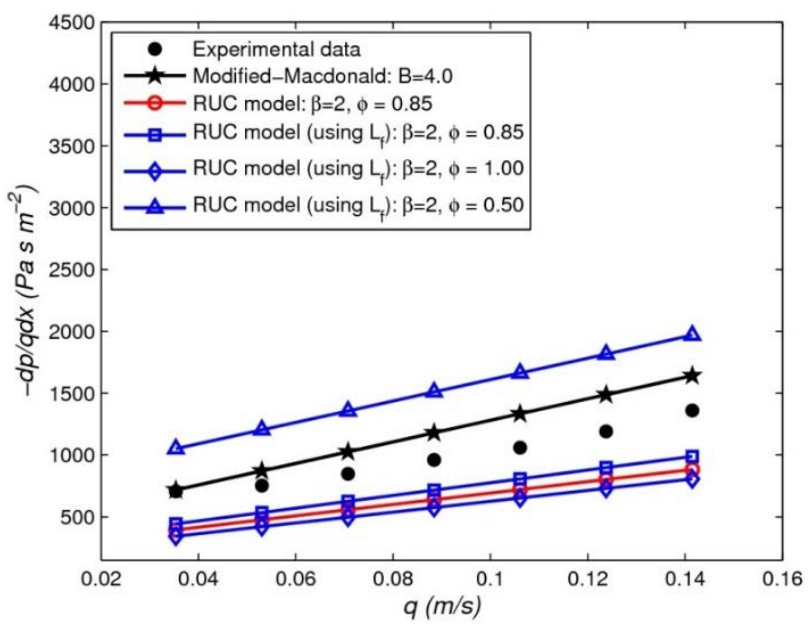

Figure 1: RUC model vs MM model for day 39.

In this study two cases were considered in the empirical modelling approach for the initial guessed coefficient $B$-values when examining the MM model, i.e. $B=2$ and $B=3$ with $\varepsilon$ set to the value of the experimentally determined porosity. After optimization, it was found that $\varepsilon$ and $B$ were independent of the initial guessed values. The same holds for when $\varepsilon$ was being optimized with the RUC model. Since the CR model is dependent on three unknowns, i.e. $\varepsilon, a_{v d}$ and $\tau$, two different combinations of initial guessed values were tested, i.e. Case 1: $\left(\tau ; a_{v d}\right)=(1.5 ; 500)$ and Case $2:\left(\tau ; a_{v d}\right)=$ (1.3; 600). The differences between the optimized $\varepsilon$-values based on the different initial guessed values, applicable to the MM, RUC and CR models, are graphically represented in Fig. 2.

Fig. 2 shows that the RUC model under-predicts the data. The MM model gives the best results when compared to the experimental data. Only one set of data points is reflected for the MM model, since it is unaffected by the initial guessed $B$-values. The most probable reason for the excellent performance of the MM model, is the fact that it was used in Excel $^{\circledR}$ to obtain the optimized value of $\varphi$. Considering the CR model, the results are less accurate when compared to those obtained by the other models. Although the differences between the porosity values based on the initial guessed values used in Case 1 and 2 are relatively small, the CR model showed to be dependent on the initial guessed values.

The percentage differences, $P D$, between the optimized porosity, SSA and tortuosity values obtained for Case 1 and 2, were determined as follows:

$$
P D=\frac{x_{1}-x_{2}}{x_{1}} \times 100
$$

where $x_{1}$ and $x_{2}$ denote the initial guessed values from Case 1 and 2, respectively. The results are given in Table 1. The rather significant values on days 57 and 71 may be a result of less accurate pressure drop measurements on these days. The values given in Table 1 reveal that the percentage difference values for each day are of the same order of magnitude. This implies that regardless of what variable is being optimized, the initial guessed value has the same effect on the result.

The sensitivity to the initial guessed values may be a result of the fact that Excel ${ }^{\circledR}$ Solver is not only slightly sensitive to the initial guessed values, but also does not necessarily give an optimal solution when instructed to adjust multiple $(>2)$ unknown variables. 


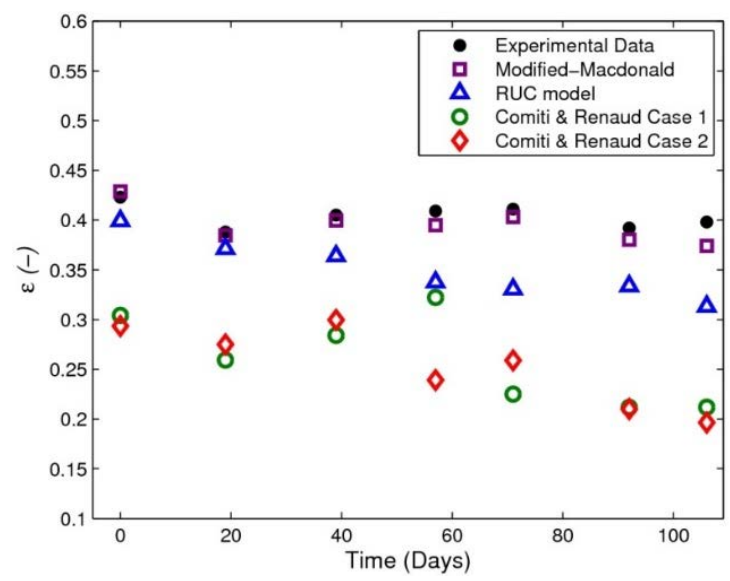

Figure 2: Comparison of the optimized and predicted porosity values with the experimental values over time.

Table 1: Percentage differences in $\varepsilon, a_{v d}$ and $\tau$ between Case 1 and Case 2.

\begin{tabular}{|l|l|l|l|}
\hline \multirow{2}{*}{ Day } & \multicolumn{3}{|l|}{$\begin{array}{l}\text { Percentage difference in porosity, SSA and } \\
\text { tortuosity between Case 1 and Case 2 results }\end{array}$} \\
\cline { 2 - 4 } & Porosity & SSA & Tortuosity \\
\hline 0 & $3.46 \%$ & $4.06 \%$ & $2.61 \%$ \\
\hline 19 & $-6.09 \%$ & $-6.80 \%$ & $-4.53 \%$ \\
\hline 39 & $-5.47 \%$ & $-6.35 \%$ & $-4.09 \%$ \\
\hline 57 & $25.79 \%$ & $28.77 \%$ & $20.05 \%$ \\
\hline 71 & -15.07 & $-16.38 \%$ & $-11.01 \%$ \\
\hline 92 & $0.76 \%$ & $0.78 \%$ & $0.56 \%$ \\
\hline 106 & $7.23 \%$ & $7.37 \%$ & $5.42 \%$ \\
\hline
\end{tabular}

The optimization results for the roughness coefficient $B$ is graphically represented in Fig. 3. It is clear that $B$ does not maintain a constant value throughout the course of the experiment based on the MM model. This is a possible result of increasing biofilm growth on the surface of the packing material and non-uniformity violating the assumption of homogeneous biofilm growth [4]. The differences between the optimized values for $\tau$ and the effects of the different initial guesses for the CR model are shown in Fig. 4. A model from Lanfrey et al. [10], i.e.:

$$
\tau=1.23 \frac{(1-\varepsilon)^{\frac{4}{3}}}{\varepsilon \varphi^{2}}
$$

was used to predict the tortuosity for both the MM and RUC models. The geometrical tortuosity, i.e.:

$$
\tau=\frac{\varepsilon}{\varphi\left(1-(1-\varepsilon)^{\frac{2}{3}}\right)},
$$

is based on the rectangular geometry of the RUC model [4]. Note that the optimized porosity values were used in both eqns (10) and (11). 
The RUC model overpredicts the tortuosity data when using the model from Lanfrey et al. [10] and under-predicts it with eqn. 11. Since the differences in the tortuosity values based on the initial guessed values of Case 1 and 2 in the CR model are negligibly small, it can be concluded that $\tau$ is unaffected by the initial guessed values. A possible cause for this might be the fact that there is a small difference, i.e. 0.2 , in the initial guessed values of $\tau$ for Case 1 and 2. Similar to the percentage difference in $\varepsilon$-values given in Table 1, the percentage difference in $\tau$-values (although negligibly small) show that there are greater differences in values on days 57 and 71 .

In Fig. 5 the predicted biofilm affected SSA values of the MM and RUC models were obtained with eqn (6) and graphically represented in Fig. 5. Both the MM and RUC models give accurate results, since the SSA values are of a similar order of magnitude when compared to the experimental data. This may be a result of the MM and RUC models only having $\leq 2$ unknown variables that had to be optimized. The CR model, however, overpredicts the data from days 0-39 and under-predicts the data from day 71 onwards.

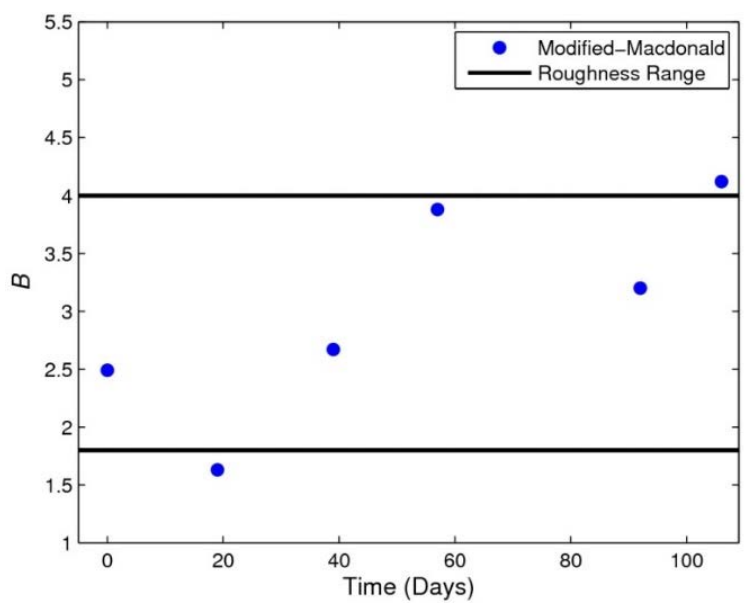

Figure 3: Optimized roughness coefficient values over time.

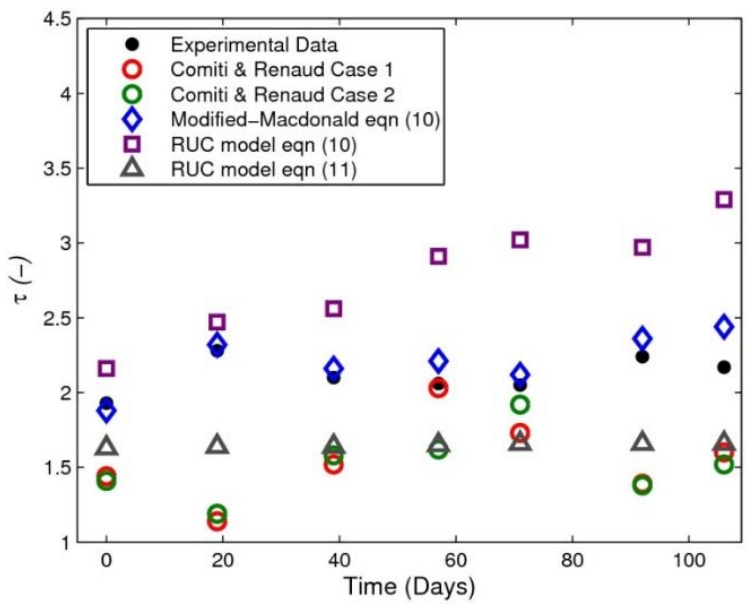

Figure 4: Comparison of the optimized and predicted tortuosity values with the experimental values over time. 


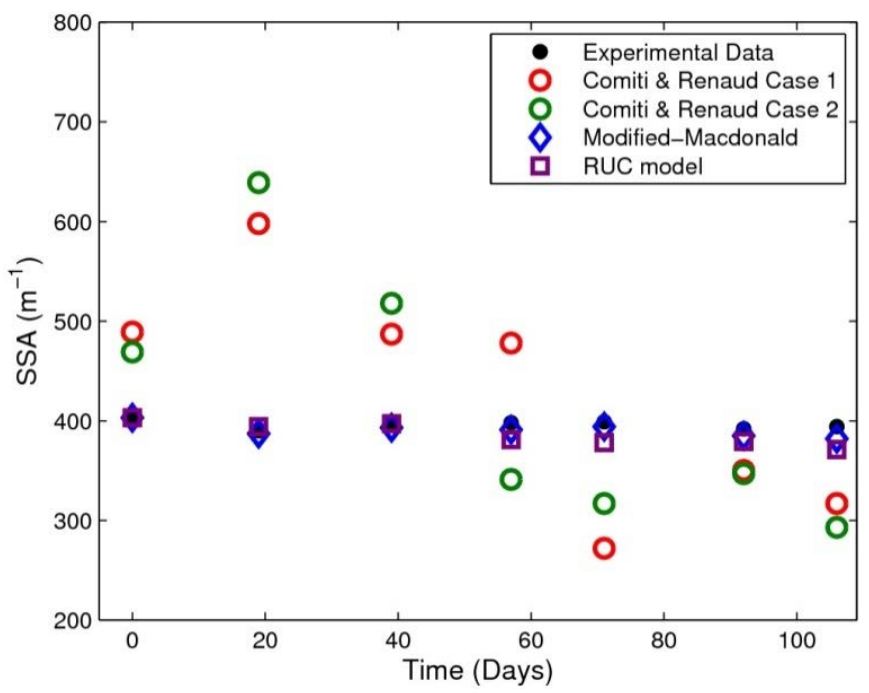

Figure 5: Comparison of the optimized and predicted SSA values with the experimental values over time.

As already mentioned, eqn (4) was used to determine $L_{f}$. The results are shown in Fig. 6. In both cases of the MM and RUC models the optimized $\varepsilon_{f}$-values were used in order to determine the biofilm thickness. Recall that the CR model was not used for the optimization of $L_{f}$ due to the previous poor performance of the model.

From Fig. 6 it is clear that both the MM and RUC models mostly overpredict the $L_{f}$ values, whilst the more accurate results were once again obtained from the MM model. A comparison of the $L_{f}$-values, obtained from the analytical and empirical approaches, is presented in Fig. 7. The $L_{f}$-values obtained from eqn. 4 gradually decreases with an increase in $\varepsilon_{f}$, as expected. The optimized $L_{f}$-values obtained by the empirical modelling approach are scattered and shows no specific trend. This is due to the alternating decreasing and increasing porosity values obtained with the MM model (as shown in Fig. 2) used to optimize the $L_{f}$-values.

It is important to note that the $L_{f}$-values are dependent on the initial porosity. After performing a sensitivity analysis on the $\varepsilon_{0}$-value in the analytical modelling approach, it is found that a 1 and $2 \%$ error in $\varepsilon_{0}$ leads to a difference of a factor of approximately $0.1 \times$ $10^{-4}$ in the value of $L_{f}$. This phenomenon is shown in Fig. 7. The accuracy of the analytical approach, in specific, therefore depends on the accuracy of the experimentally measured $\varepsilon_{0}$-values.

Fig. 8 shows the biofilm affected SSA as a function of biofilm thickness. Each data point of the analytical model prediction represents the average of the SSA values obtained from the seven different pressure drop measurements (eqn (7)) on a specific day.

The scatter of data in Fig. 8 is a result of the fluctuation in measured pressure drop values as well as the average calculation. The biofilm affected SSA values obtained from the empirical modelling approach stays more or less constant. This is due to the large order of magnitude difference between the terms containing $L_{f}$ in eqn. 6 and the terms excluding $L_{f}$. 


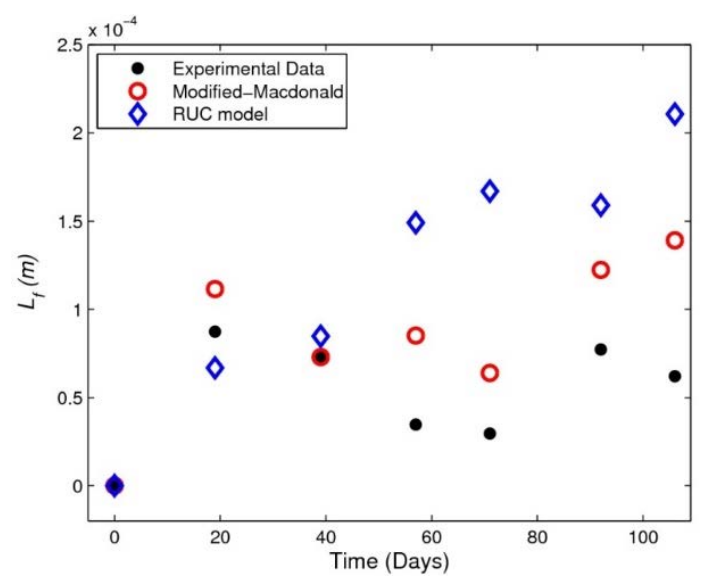

Figure 6: Comparison of the optimized and predicted biofilm thickness values with the experimental values over time.

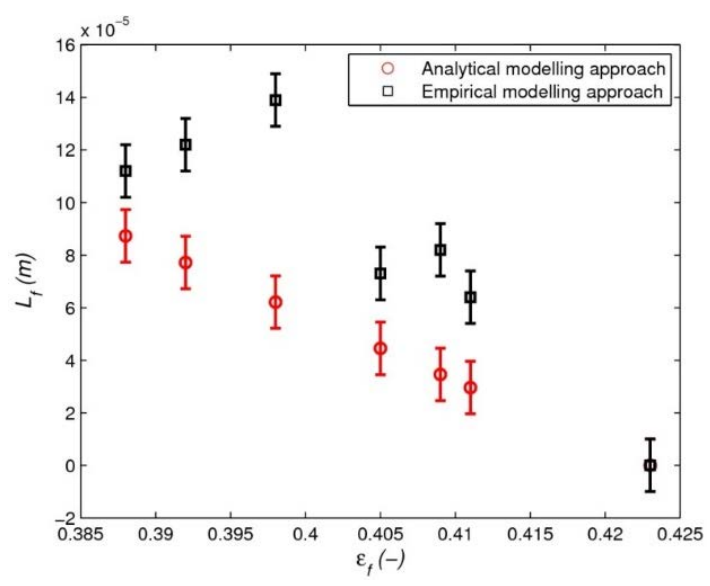

Figure 7: Biofilm thickness as a function of biofilm affected porosity.

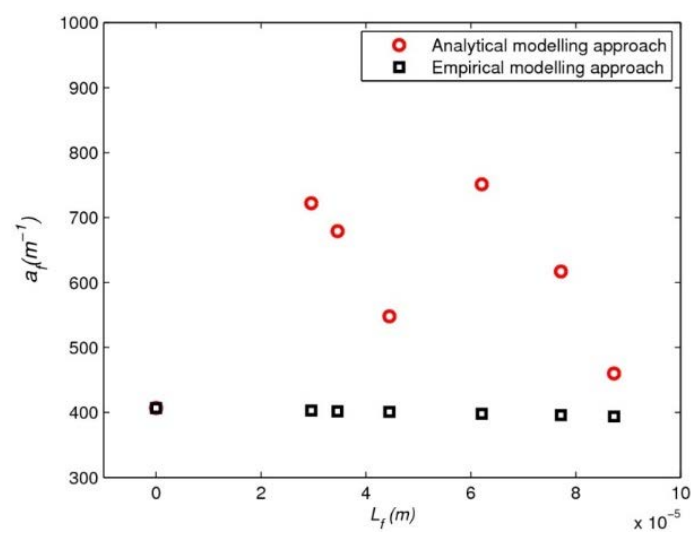

Figure 8: Biofilm affected SSA as a function of biofilm thickness. 


\section{CONCLUSIONS}

After comparing the optimized porosity values obtained from the three models, with the measured porosity values, it was found that the CR model cannot be used to predict the porosity of a packed bed. This may be a result of the fact that Excel ${ }^{\circledR}$ Solver is not only slightly sensitive to the initial guessed values, but also does not necessarily give an optimal solution when instructed to adjust multiple $(>2)$ unknown variables. The CR model was therefore excluded from predicting biofilm thickness values. The MM model overall gave remarkable results when compared to the experimental values and a probable cause is due to the fact that the MM model was used in Excel ${ }^{\circledR}$ to obtain the value of the sphericity. Although the results obtained with the analytical modelling approach are less accurate than those obtained with the empirical modelling approach, the former method is justifiable from a physical point of view. The RUC model also does not contain any empirical curve fitting parameters such as the coefficients of 180 and 1.8 in the MM model. The outcome in terms of model performance between the MM and RUC models may, however, be different should the RUC model be used to optimize the sphericity value in the empirical modelling approach instead of the MM model. This will be investigated in future studies. The performance of the porous media models not only depends on which model was used to obtain the sphericity value, but also on the number of unknown variables which directly influences the optimization results. A major advantage of the analytical modelling approach is that the pressure drop can alternatively be used to predict the biofilm affected SSA values by making use of the experimentally measured porosity values. When applying the empirical approach, one is not able to predict the pressure drop values, since it is already used in the sum of the squared residuals in order to obtain the optimized values.

\section{ACKNOWLEDGEMENTS}

The first author wishes to thank the DST-NRF Centre of Excellence in Mathematical and Statistical Sciences (CoE-MaSS) for funding. Opinions expressed and conclusions arrived at are those of the authors and are not necessarily to be attributed to the CoE-MaSS.

\section{REFERENCES}

[1] Alonso, C. et al., Gas treatment in trickle-bed biofilters: Biomass, how much is enough? Biotechnology and Bioengineering, 54, pp. 583-594, 1997.

[2] Dumont, E., Guzman, L.M.A., Susa, M.S.R. \& Andres, Y., $\mathrm{H}_{2} \mathrm{~S}$ biofiltration using expanded schist as packing material: Performance evaluation and packed-bed tortuosity assessment. Journal of Chemical Technology and Biotechnology, 87, pp. $725-731,2012$.

[3] Manure management and air quality: Biofilter design information; University of Minnesota Extension, Office of the Dean, 240 Coffey Hall, Online. www.extension. umn.edu/agriculture/manure-management-and-air-quality/air-quality/biofilter-designinformation/. Accessed on: 27 Jan. 2017.

[4] Dumont, E., Woudberg, S. \& Van Jaarsveld, J., Assessment of porosity and biofilm thickness in packed beds using porous media models. Powder Technology, 303, pp. 76-89, 2016.

[5] Woudberg, S., Van Jaarsveld, J. \& Dumont, E., Analytical determination of the effect of biofilm growth on the pressure drop over a biofilter. Proceedings of the World Congress on Momentum, Heat and Mass Transfer, Prague, Czech Republic, April 4-5, 2016. 
[6] Morgan-Sagastume, F., Sleep, B.E. \& Allen, D.G., Effects of Biomass Growth on Gas Pressure Drop in Biofilters. Journal of Environmental Engineering, 127, pp. 388-396, 2001.

[7] Comiti, J. \& Renaud, M., A new model for determining mean structure parameters of fixed beds from pressure drop measurements: application to beds packed with parallelepipedal particles. Chemical Engineering Science, 44, pp. 1539-1545, 1989.

[8] Du Plessis, J.P. \& Woudberg, S., Pore-scale derivation of the Ergun equation to enhance its adaptability and generalization. Chemical Engineering Science, 63, pp. 2576-2586, 1990.

[9] Macdonald, I.F., El-Sayed, M.S., Mow, K. \& Dullien, F.A.L., Flow through Porous Media-the Ergun Equation Revisited. Industrial and Engineering Chemistry Fundamentals, 18, pp. 199-208, 1997.

[10] Lanfrey, P.Y., Kuzeljevic, Z.V. \& Dudukovic, M.P., Tortuosity model for fixed beds randomly packed with identical particles. Chemical Engineering Science, 65, pp. 1891-1896, 2010. 